\title{
Astrobiology as a NASA Grand Challenge
}

Co-authors:

Shawn Domagal-Goldman, NASA Goddard Space Flight Center, shawn.goldman@nasa.gov

Giada Arney, NASA Goddard Space Flight Center, giada.n.arney@nasa.gov

Eric D. Lopez, NASA Goddard Space Flight Center, Eric.D.Lopez@nasa.gov

Co-signers:

Kimberly Bott (UCR)

Chuanfei Dong, Princeton University, dcfy@princeton.edu

Niki Parenteau, NASA Ames Research Center, mary.n.parenteau@nasa.gov

Victoria Meadows, University of Washington, meadows@uw.edu

Britney Schmidt, Georgia Tech, britneys@eas.gatech.edu

Timothy Holt, University of Southern Queensland, timothy.holt@usq.edu.au

Stephen Kane, University of California, Riverside, skane@ucr.edu

Laura Schaefer, Stanford University, Ikschaef@stanford.edu

Aki Roberge, NASA GSFC, aki.roberge@nasa.gov

Thomas Beatty, University of Arizona, tgbeatty@arizona.edu

Ravi kumar Kopparapu, NASA Goddard Space Flight Center, ravikumar.kopparapu@nasa.gov

Ehsan Gharib-Nezhad (NASA ARC)

Der-you Kao, University of Maryland/NASA GSFC,der-you.kao@nasa.gov

Monica Vidaurri, Howard University/NASA GSFC

Andrew Vanderburg, University of Wisconsin-Madison

Chuhong Mai (Arizona State University)

Chester 'Sonny' Harman, NASA Ames Research Center, sonny.harman@nasa.gov

Jessica Noviello, Arizona State University

Theresa Fisher, Arizona State University, tfisher4@asu.edu

Eric T. Wolf, University of Colorado, Boulder, eric.wolf@colorado.edu

Ashley Herbst, Arizona State University

M.J. Way, NASA Goddard Institute for Space Studies, michael.way@nasa.gov

Jonathan Fortney, University of California, Santa Cruz, jfortney@ucsc.edu

Ryan Felton, Catholic University of America, ryan.felton@nasa.gov

Hilairy E. Hartnett, Arizona State University, h.hartnett@asu.edu

Dawn M. Gelino, NASA Exoplanet Science Institute, IPAC/Caltech

Anneliese Ardizzone, GSFC, rockettome@yahoo.com

Jaime Crouse, GSFC, jaimescrouse@gmail.com

Paul K. Byrne, North Carolina State University, paul.byrne@ncsu.edu

Sandra Bastelberger, University of Maryland, sandra.t.bastelberger@nasa.gov

Stephanie Olson, Purdue University, stephanieolson@purdue.edu

Vinayak Shastri, University of St Andrews, vs72@st-andrews.ac.uk

Sarah E. Moran, JHU 


\begin{abstract}
"Are we alone" is a question whose ambition can only be met with a NASA-led global collaboration. In this white paper, we describe how this makes "The Search for Life Beyond Earth" a new Grand Challenge for NASA. As described by the White House Office of Science and Technology Policy and the White House National Economic Council, Grand Challenges are "ambitious but achievable goals that harness science, technology, and innovation to solve important national or global problems and that have the potential to capture the public's imagination." NASA had identified an "Asteroid Grand Challenge" centered on the Asteroid Retrieval Mission, which was closed out in June, 2017. NASA also identified the "Urban Air Mobility" as a grand challenge in March of 2020. The Artemis Program also contains the qualities of an agency-level Grand Challenge. Here, we explain how NASA's next Grand Challenge could be focused on "The Search for Life Beyond Earth," with multiple Astrophysics and Planetary Science missions as its centerpiece.
\end{abstract}

\title{
Introduction
}

Our generation has been presented with a unique opportunity: to be the first humans to comprehensively search for signs of life in and beyond our Solar System. This is possible because of the progress of astrobiological research, the identification of habitable environments inside the Solar System, the discovery that there are potentially habitable worlds orbiting other suns, the creation of an interdisciplinary research community, and the development of technologies that enable missions designed to search for signs of life on worlds in and beyond the Solar System. If the Astro2020 and Planetary Decadal Surveys decide to capitalize on this opportunity by prioritizing ambitious astrobiology missions, it will set the stage for a global collaboration that revolutionizes our understanding of humanity's place in the cosmos.

This comprehensive search for life is ideal for a Grand Challenge. This program would be inarguably ambitious, yet achievable by projects started in the era (2023-2032) covered by the Planetary Science and Astrobiology Decadal Survey. It would harness science, technology, and innovation to solve important problems pursued across the globe; and it would present an unmatched ability to capture the public's imagination. These qualities are the top-level criteria for a Grand Challenge, as defined by the United States Office of Science and Technology Policy.

In this White Paper, we propose such a Grand Challenge. First, we briefly review the topics covered by astrobiology researchers. Then, we introduce the requirements for an agency-level Grand Challenge and explore how "Astrobiology and the Search for Life" meets the requirements for a NASA Grand Challenge, including a partial list of ongoing activities that could serve as a foundation for a broader program. Finally, we close with the relationship of this Grand Challenge to the projects being submitted for consideration for the NAS Planetary Science and Astrobiology Decadal Survey 2023-2032.

\section{Review of Astrobiology:}

Astrobiology is much more than "the search for life beyond Earth." Instead, it is a discipline that also develops our capability to conduct and understand the results from a search for life elsewhere. These improvements result from a broad set of interdisciplinary investigations across the globe. The NASA 2015 Astrobiology Roadmap outlines current research on Astrobiology as covering the following topics:

1. Identifying abiotic sources of organic compounds 
2. Synthesis and function of macromolecules in the origin of life

3. Early life and increasing complexity

4. Co-evolution of life and the physical environment

5. Identifying, exploring, and characterizing environments for habitability and biosignatures

6. Constructing habitable worlds

7. Challenges and opportunities in astrobiology

Of these 7 topics, only 5 . is directly related to the search for life; the other 6 provide the necessary planetary and system context and theoretical framework to that search. By identifying the abiotic sources of organic compounds, and by studying the synthesis and function of macromolecules in the origin of life, we improve our ability to know how life arose on Earth and how/where it might arise elsewhere. By studying early life, increasing complexity, and the co-evolution of life and its physical environment, we gain knowledge on issues related to habitability, broaden the biosignatures we can search for, and improve our understanding of life as a planetary process. By exploring how habitable worlds are constructed, we help focus our search for life on the most likely locations, which is critical when designing missions and instruments that have constrained budgets for cost, mass, power, volume, or other parameters. Finally, by understanding the challenges and opportunities faced by individuals and the field as a whole, we improve our capability to conduct astrobiology research and missions.

\section{Astrobiology as a Grand Challenge}

Each of the research themes outlined above demands an interdisciplinary approach that engages a wide variety of expertise and input. This interdisciplinary nature drives the field towards collaborations that reach across disciplines, funding stovepipes, and national borders. The technical challenges faced by missions robustly capable of identifying signs of life on other worlds demands spaceflight partnerships. The breadth of skills required by this search will engage most of the expertise at NASA, while growing connections to external partners and inspiring people across the globe. This is what makes Astrobiology and The Search for Life a perfect fit for a NASA Grand Challenge. The NASA Office of the Chief Technologist described the following criteria for a NASA Grand Challenge:

- Clearly relate to NASA's mission and strategy.

- Unify and inspire public and private constituencies around a clear "call to action". It must be specific enough to convey success criteria and broad enough to capture the imagination of participants and the public.

- Spur significant developments in a wide range of domains vital to the nation and the world.

- Serve as a beacon for high-impact, trans-disciplinary collaborations among the government, industry companies, universities, non-profits, philanthropists, and individuals including scientists and engineers. Given NASA's investments, partnerships and ability to leverage activities and resources, the grand challenge must be ambitious, but realistically achievable within $10-20$ years.

Below, we discuss how these requirements are met by Astrobiology and the Search for Life, by detailing how this Grand Challenge would radiate out from the core areas of expertise in Astrophysics and Planetary Science to encompass and include expertise from across the Science Mission Directorate (SMD), the Agency, and both traditional and non-traditional global partners.

\section{Relationship to NASA's Mission and Strategy:}


As described in the 2018 NASA Strategic Plan, the Mission of the agency is to: Lead an innovative and sustainable program of exploration with commercial and international partners to enable human exploration across the Solar System and bring new knowledge and opportunities back to Earth, support growth of the nation's economy in space and aeronautics, increase understanding of the Universe, and our place in it, work with industry to improve America's aerospace technologies, and advance American leadership.

A search for life is responsive to this Strategy and Mission. The search for life would necessitate research, technology development, and mission programs that are each innovative, and sustainable. As we will explain in more detail below, it would involve both commercial and international partners. And it would both benefit from and help give extra meaning to human exploration of the Solar System. Astrobiology research on the co-evolution of life and its host environment, and the results from missions that search for signs of global biospheres on potentially habitable planets both improve our understanding of how our home planet operates. And astrobiology research and missions would increase our understanding of the Universe and our place in it. All of this would be done in a way that supports growth of the nation's economy in space, would improve America's aerospace technologies, and would be an extremely visible example of American leadership.

\section{Astrobiology Missions at the Center of the Grand Challenge}

The core of this Grand Challenge will be missions designed to search for signs of life and habitable environments beyond Earth. This search would be conducted in the Solar System as well as on exoplanets. In the solar system, it would consist of a fleet of orbiters and landers to multiple targets, that would detail the history of the Solar System's set of habitable environments. These searches for habitable conditions and signs of life across different targets would also help unite the fields of astrobiology and comparative planetology, and answer more detailed questions than the top-level question of "Are we alone?" Searches for life on Titan would be relevant to the hypothesis that water is a fundamental requirement for life. The related hypothesis of "if water, therefore life" could be tested by a search for life across multiple ocean worlds. The search for past and present life on Mars could help tell the story of a once-living biosphere. And the study of the climate histories of Mars and Venus help us understand how a planet can lose potentially habitable conditions. Finally, sample return missions from Mars and from ocean worlds present us with one of the fastest-track paths to a search for life that includes a rigorous set of measurements.

This astrobiological fleet would be complemented and augmented by the missions that are being separately and simultaneously considered by the Astrophysics 2020 Decadal Survey. Most notably, the LUVOIR, HabEx, and Origins mission concepts all strive to detect biosignatures as a key science case, and for each concept that goal had a driving influence on architecture decisions. Additionally, these missions - as well as the Lynx mission concept also being submitted for consideration by Astro2020 - would conduct observations related to the other areas of astrobiology outlined above, in particular our understanding of how habitable worlds are constructed. Finally, their ability to monitor Solar System targets would also allow these missions to play a support role to the fleet of vehicles mentioned above.

\section{The Astrobiology Grand Challenge as a NASA-wide Initiative}


This work requires the coordination of research and mission observations from across the NASA Science Mission Directorate (SMD) portfolio. In addition to the roles for Astrophysics and Planetary Sciences, there are specific inputs that are essential from Heliophysics and Earth sciences. Heliophysics research and missions inform our understanding of atmospheric loss, which is a controlling process for the habitability of exoplanets, and the past habitability of Mars. Earth sciences tools - in particular climate and chemistry models - have been used to explore the co-evolution of life with the physical environment throughout Earth history, to study the past habitability of Solar System bodies, and model the potential habitability of exoplanets.

Each NASA SMD Division stands to benefit from the public and stakeholder interest in the search for life. This is particularly true for the missions that would have an impact on this search. And some missions may have indirect or unexpected contributions to Astrobiology; for example, the full-size images of Earth from the Deep Space Climate Observatory (DSCOVR) have been used to simulate future exoplanet direct imaging data. Additionally, there are tools and community standards that have been developed by these fields that could benefit the other divisions and their communities, as cross-division collaboration increases. An early example of this is the creation of the Exoplanet Modeling and Analysis Center - a NASA-hosted library of community models for exoplanet - which was inspired and modeled after the Community Coordinated Modeling Center funded by the Heliophysics division. Lastly, the application of tools to simulate planetary processes on a wider variety of targets will improve their validation. For example, the application of climate models to the study of the habitability of Mars and exoplanets expands the physics of these tools as it produces evidence against the argument that such models are "fine-tuned" to the Earth or to studies of anthropogenic climate change.

Beyond SMD, humans and robots in space can expand and extend the capabilities of astrobiology missions - including the four flagship concepts being studied for Astro2020. In-space assembly would allow for the construction of larger, more capable telescopes than would otherwise be possible with telescopes that require a single launch vehicle. Additionally, the servicing of existing missions would allow for additional generations of instruments to follow-up on initial surveys. There is likely to be considerable debate within the science community about the first claimed signs of life from an exoplanet; serviceability would allow scientists to design and operate a second generation of instruments designed to return data that would help resolve such debates. And the extension of observations would enable science cases that would otherwise be implausible or impossible. This could include the observations of additional transits of potentially habitable exoplanets to increase the signal/noise ratio of spectra from such worlds, or the measurement of season-/latitude-dependent features of reflected light from an exoplanet. The latter observation is particularly expensive, but could yield compelling results such as the first low-resolution map of an ocean-bearing exoplanet.

Additionally, the humans/robots that explore the Solar System would bring back samples that have tremendous astrobiological value. In the near-term, astronauts that land on the moon can search for samples of Archean or Hadean Earth (Earth meteorites that have landed on the moon). Studies of these eons in Earth history have played a major role in astrobiological research on the co-evolution of life and its physical environment. However, geological processes have destroyed much of the Earth's rock record from this time, and those samples that have survived have often been altered. Therefore, any pristine Archean/Hadean Earth samples returned by astronauts would have tremendous value. Samples of the moon itself would also bring value, as they could undergo precise absolute dating that would help uncover the history 
of the Moon. By tying this dating to the craters on the Moon, this would also improve our dating of many surfaces throughout the Solar System, which primarily rely on cratering records. Similarly, samples returned from comets and asteroids would yield insights on the delivery of abiotic sources of organic compounds from such bodies, and on how habitable worlds are constructed. Multiple missions are ongoing or have been proposed to return such samples; humans could participate in this endeavor when they visit such bodies.

The human exploration of Mars would bring about astrobiological revolutions on its own. The presence of humans on Mars, or even in cis-Mars space, would dramatically expand our capability to conduct research on Martian history. Real-time operation of rovers could improve their efficiency, and the communications relays and weather stations required to support a human presence would return their own data on the Martian environment. Perhaps most importantly, humans could bring samples from Mars; a long-term goal of the Mars Exploration Program. A rover designed to select and cache samples for return is the main driver of the number one recommendation from the last NAS Planetary Science Decadal Survey Report, "Visions and Voyages for Planetary Science in the Decade 2013-2022" a Mars sample return mission will be considered by this Decadal Survey.

The incorporation of astrobiology science into human exploration of the Solar System could bring benefits to human spaceflight. The public interest in the science of astrobiology could bring an additional rationale for the support of such a program, and a greater level of overall interest in the science conducted by astronauts. The links between the Hubble Space Telescope and the human servicing missions raised the profile of both endeavors, and served as a model for how human spaceflight and science could both benefit from collaboration. On a future human mission to Mars, a mass spectrometer could look for potential biosignatures, or be used to track the bone density of astronauts. Scientists and explorers may search for the same exact data but for different reasons: weather/dust storms on Mars, life in special regions, and methane/water on Mars are all topics that generate tremendous astrobiological or planetary science interest, and are of independent interest due to their relationship to resources and hazards to astronauts.

\section{The Astrobiology Grand Challenge as a Global Endeavor}

Astrobiology is inherently a global endeavor, both by practical necessity and by moral imperative. The search for life is something that will change our entire planet's view of life in the cosmos, and as such it should engage and include global participation. Such participation will be required. The most ambitious astrobiology missions would be enabled or enhanced by leveraging skillsets, industries, and funding offered by nations other than the United States. Further, NASA, NSF, DOE, and other global space and science agencies should work to ensure their mission complement - and to not compete with - one another. While this paper focuses on US-led/NASA-led missions due to it being a submission to the US National Academy of Sciences white paper call, there are many missions to planetary targets near and far that would be an essential part of the endeavor. To make this a NASA Grand Challenge, the agency should lead some of the ambitious missions described below, include the expertise of our international partners on those missions, and also participate in the missions led by other nations.

NASA is already partnered with a variety of research institutions - both in the US and around the globe - in the pursuit of astrobiology research. Through the NASA Astrobiology Institute (NAI), numerous teams - including many led by universities - have advanced astrobiology research since the foundation of the NAI in 1998. Newly created astrobiology 
"research coordination networks" will expand and continue these partnerships with members at numerous NASA centers and universities.

Partnerships with other agencies would also be required for this Grand Challenge. Existing, extensive partnerships already exist between NASA and NSF related to research on the synthesis and function of macromolecules in the origin of life, searches for meteorites in Antarctica, and research in extreme environments around the globe, drilling programs for samples of astrobiological interest, and the testing/development of instruments and mission operations in analogue environments. Such partnerships could be strengthened or expanded. Additional partnerships could arise with NIH or NSF for the exploration of the development of complexity and multicellularity or on the understanding of how life operates in extreme environments. And the search for life on exoplanets will be best accomplished with coordinated observations from both space-based and ground telescopes. These different observatories have complementary wavelength ranges, utilize different observational techniques, and would target planets around different star types. Ultimately, in order to produce a comprehensive understanding of how life and habitability is a function of the stellar environment, both groundand space-based observatories will be required.

To truly expand astrobiology research into an agency-level Grand Challenge, multiple missions would be required. These missions would be ambitious in intent, and would lead to equally ambitious technical requirements that demand collaboration. To make such missions happen, NASA has always engaged industry and international partners. In this case, new partnerships would form that could improve these missions and our ability to assess their data.

The most obvious link to external partners is to the aerospace industry that has been a historical partner on NASA/SMD missions. This partnership has enabled missions large and small for decades, and would continue and be absolutely central to an Astrobiology Grand Challenge. The expertise on every phase of the mission lifecycle, and the experience these partners bring from beyond NASA would enable the endeavor. Details on specific technical challenges are present in the White Papers that will be submitted to Astro2020, and eventually to the Decadal Surveys for Planetary Sciences and Heliophysics. Most of these technical challenges will require a tight partnership with industry to ensure success, and we refer you to those separate white papers for details. As with the benefits to SMD and HEOMD, there would be benefits to industry that go beyond financial incentive. Their participation or leadership of any astrobiological endeavor will bring with it positive public notoriety. It also can provide opportunities to advance technological or workforce capabilities, as the specific technical challenges presented by each mission is overcome.

Partnerships with private entities, which have previously been mostly limited to building spaceflight hardware, must expand and could include development of generic research tools, new codes, and new methodologies to analyze and interpret data. Private participation in astrobiology endeavors is already increasing. One example is the NASA Frontiers Development Lab (FDL) which provides another model for partnerships with industry. The FDL has brought together researchers from across the globe to work on space science problems funded by NASA, through the use of techniques/tools/expertise provided by experts on artificial intelligence (AI) and machine learning (ML). In 2018, FDL expanded to include its first astrobiology studies. These teams combined NASA-funded models of exoplanet atmospheric chemistry and spectroscopy with $\mathrm{Al} / \mathrm{ML}$ tools to create tools that can retrieve compositional data from spectra of exoplanets. This is one example of the myriad problems that can be addressed with $\mathrm{Al} / \mathrm{ML}$. 
Non-traditional partners would also be fully involved in the search for life. The difficulty of the endeavor, the broad public interest in its results, and the societal implications of the search all provide a drive towards the incorporation of diverse skill sets and perspectives. This should lead to a diverse global community that is included in the Grand Challenge. Successes along these lines exist and could be expanded upon. The Astrobiology Program has created multiple collaborations between scientists, philosophers, and religious scholars to understand the societal impacts that would result from the discovery of life beyond Earth. It also created the Baruch S. Blumberg Chair of Astrobiology at the Library of Congress for "research at the intersection of the science of astrobiology and its humanistic and societal implications." And it is pursuing partnerships with the Navajo Nation and prisons in the United States that open scientific conversations with groups that are far too often left out of scientific discourse.

The above is a very partial list of partnerships within and beyond the agency that demonstrate the broad impact astrobiology could have on the world, and on the engagement the global community should have on this search. Because the search for life is inherently engaging, it can lead to developments in a wide range of domains including but not limited to astronomy, planetary science, Earth sciences, heliophysics, human exploration, aerospace engineering, multicultural research, science communication, space ethics, and computer science. To fully succeed in discovering habitable and inhabited worlds elsewhere, we suggest that the teams that work on these missions must be equally diverse. In addition to incorporating the expertise listed above, these teams must also be inclusive and representative of diverse areas of science, combining the expertise of astronomers, planetary scientists, origins of life researchers, biologists, geologists, atmospheric scientists, and others. There are myriad challenges our entire community faces to realize that goal - we anticipate separate white papers on this topic; following their suggestions is foundational to an Astrobiology Grand Challenge.

\section{The Astrobiology Grand Challenge and NASA Flagship Concepts for Astro2020}

The search for life beyond Earth is deeply interdisciplinary, and the missions required to discover life on other worlds will require partnerships beyond NASA and the academy to include private enterprise as part of a trans-disciplinary endeavor. The pursuit of any "wicked problem" requires this trans-disciplinary approach; previous efforts at this scale, for example Apollo, have had impacts reaching far beyond the original mission concept. Many of the missions that will be considered have direct and obvious connections to the search for habitable environments and life, due to their pursuit of those goals. Other missions that study the origins of planets, the delivery of volatiles to those worlds, and the associated emergence of habitable conditions also play a role in the telling of this story. And studies of uninhabitable planets, such as the gas and ice giants in the outer reaches of the Solar System, have relevance both for their ability to improve our understanding of planetary processes via comparative planetology, and also due to their ability to act as analogues and context for exoplanets where this search will occur in less detail, but with significantly greater sample sizes.

This suite of mission concepts could serve as the catalyst for and central component of a new Grand Challenge for NASA. All manner of expertise within and beyond the agency would be required. And the ambitious goal - to determine whether or not humanity is alone in the Universe - could lead to NASA's next "Apollo moment." 